\title{
MARCADORES TUMORAIS NO CÂNCER COLORRETAL
}

\author{
TUMOR MARKERS IN COLORECTAL CANCER
}

\author{
Luís César Fernandes, ACBC-SP ${ }^{1}$ \\ Delcio Matos ${ }^{2}$
}

\section{INTRODUÇÃO}

As neoplasias colorretais constituem a terceira neoplasia em incidência em variadas populações ${ }^{1}$. Representam importante letalidade, configurando de $10 \%$ a $20 \%$ de todos os óbitos motivados por neoplasias; o índice de sobrevida geral sendo inferior a $40 \%{ }^{2,3}$.

Apesar dos aperfeiçoamentos no diagnóstico e no tratamento do câncer colorretal, as taxas persistem quase inalteradas, com discreta redução nos índices de mortalidade $^{1,4}$.

Nas tentativas de estabelecimento de diagnóstico precoce e melhor tratamento destacam-se os marcadores tumorais, produtos moleculares secretados por tecido neoplásico, detectáveis em células e fluidos orgânicos. São capazes de indicar presença, extensão, resposta ao tratamento e presença de recorrência da neoplasia ${ }^{5}$. Podem ser hormônios, ácidos nucléicos, poliaminas, antígenos, enzimas, proteínas e lipídios específicos da membrana celular.

\section{Marcadores Plasmáticos e Teciduais}

Houve certa alteração no conceito de marcador tumoral. Anteriormente, considerava-se como "marcador" qualquer substância capaz de ser detectada em soro periférico de determinado paciente possuidor de neoplasia. Atualmente, componentes teciduais, inclusive genéticos, são marcadores capazes de definir diferentes prognósticos e distintas condutas terapêuticas para cada caso.

Perceba-se que marcadores teciduais não têm a capacidade que os plasmáticos possuem de determinar diagnóstico ou de indicar recidiva do processo tumoral. Limitam-se a determinar estadiamento, prognóstico e possível resposta terapêutica para determinada lesão.

\section{Marcadores no Câncer Colorretal \\ a) CEA}

Descoberto na década de 1960, determinado em amostras de soro periférico, constitui o marcador mais utilizado na prática clínica nas neoplasias colorretais ${ }^{6,7}$. Pouco efetivo no diagnóstico do câncer colorretal, por sua baixa sensibilidade em lesões iniciais, tem utilidade no estabelecimento de índices prognósticos ${ }^{8,9}$ e destaque no seguimento dos pacientes após tratamento cirúrgico pretensamente curativo, promovendo por vezes detecção precoce de recidiva tumoral ${ }^{10-13}$.

Sua sensibilidade nas etapas de diagnóstico da lesão situa-se entre $20 \%$ e $40 \%^{14}$; no entanto, sua sensibilidade no seguimento pós-operatório dos pacientes, para detecção de recidivas locorregionais ou hepáticas, pode ultrapassar $90 \%{ }^{15}$.

\section{b) CA 242, CA 19-9, CA72-4}

Tais marcadores são diagnosticados por anticorpos monoclonais específicos. Apesar de sua origem posterior ao CEA e conceito aperfeiçoado não proporcionaram melhores resultados. Sua sensibilidade no diagnóstico do câncer colorretal oscila em taxas de $30 \%{ }^{16,17}$, inferiores às do antígeno carcinoembrionário que apresenta sensibilidade em torno de $40 \%{ }^{18,19}$. Têm utilização em amostras séricas associados ao CEA; nesta condição atingem sensibilidade de aproximadamente $50 \%$ a $60 \%$, com certo valor na determinação de índices prognósticos e detecção de recidivas tumorais ${ }^{19-23}$.

\section{c) Citoqueratinas}

São constituintes da arquitetura estrutural da membrana celular, estando aumentadas em soro periférico na vigência de processos neoplásicos e sendo passíveis

1. Cirurgião-geral e Mestre pela Disciplina de Gastroenterologia Cirúrgica da Universidade Federal de São Paulo -Escola Paulista de Medicina (UNIFESP - EPM).

2. Professor Adjunto Livre-docente da Disciplina de Gastroenterologia Cirúrgica da UNIFESP - EPM.

Recebido em 05/09/2000.

Aceito para publicação em 02/10/2001.

Trabalho realizado na Disciplina de Gastroenterologia Cirúrgica do Departamento de Cirurgia da Universidade Federal de São Paulo - Escola Paulista de Medicina (UNIFESP-EPM). 
de detecção por anticorpos monoclonais ${ }^{24}$. O TPA (tissue polypeptide antigen) constituiu a citoqueratina de identificação inicial, em $1978{ }^{25}$. Um reagente mais específico (TPS - tissue polypeptide-specific antigen) foi desenvolvido em 1992, identificando o epítopo M3 da molécula TPA; um reagente atual, TPM (ou TPA-M - tissue polypeptide monoclonal antigen), descrito em 1994, dirigese a três epítopos distintos das células neoplásicas, obtendo especificidade ainda maior ${ }^{26,27}$.

Sua sensibilidade geral oscila entre $60 \%$ e $70 \%$ no diagnóstico do adenocarcinoma colorretal ${ }^{28-30}$. Tem razoável especificidade para processos neoplásicos; no entanto, positiva-se em tumores de outros sítios, tais como ovário, pulmão, próstata e mama ${ }^{26}$. Estudos sobre citoqueratinas como marcadores encontram-se em fase de desenvolvimento inicial.

\section{d) $\mathbf{p 5 3}$}

Na existência de defeitos genéticos celulares, o gene p53 pode promover o retardo da divisão celular para permitir o reparo dos genes ou pode induzir a ocorrência de apoptose (morte celular programada), na vigência de defeitos não passíveis de correção ${ }^{31}$. Alteração no gene p53 possibilita a divisão de células com imperfeições genéticas e o surgimento de neoplasias colorretais ${ }^{32}$.

O gene $\mathrm{p} 53$ origina, em condições normais, uma proteína p53 de duração efêmera tecidual. O gene p53 alterado produz uma proteína p53 alterada, de duração permanente ${ }^{33}$. Esta duração permanente possibilita a formação de anticorpos anti-p53 $3^{34}$.

$\mathrm{O}$ gene p53 alterado pode ser identificado, portanto, por três métodos: reações de PCR, determinando diretamente a alteração genética; reação imuno-histoquímica, demonstrando na análise histológica a presença da proteína p53 alterada; e dosagem em amostras de sangue periférico, identificando anticorpos contra a proteína alterada $\mathrm{p} 53^{35}$.

O marcador, apesar de promissor, apresenta deficiências conceituais e decorrentes de variantes biológicas imprevisíveis. Há neoplasias colorretais provocadas por alterações do gene p53; porém, há tumores motivados por outras anormalidades (gen $c$-Myc, $k$-ras p.ex. $)^{5,36}$, que não seriam, portanto, detectados por análises de tal marcador. Além disso, a formação de anticorpos contra a proteína alterada p53 é inconstante; a dosagem de tais anticorpos em soro periférico em estudos atuais é variável e obteve sensibilidade inferior a $30 \%$ 35,37,38. Pesquisas devem ser efetuadas para minorar as dificuldades originais do método.

\section{e) VEGF}

Ou "fator de crescimento vascular endotelial" (vascular endothelial growth factor). As neoplasias, em seu processo de crescimento, secretam substâncias estimulantes do crescimento vascular que, por sua vez, originam vasos sangüíneos responsáveis pela nutrição dos tecidos tumorais ${ }^{39}$. Tais substâncias são passíveis de detecção em soro periférico ${ }^{40,41}$. Não serão, no entanto, específicas para tumores de cólon e de reto, pelo fato de a produção do
VEGF ocorrer em diversos processos neoplásicos; encontra-se, em investigação inicial, com sensibilidade na etapa de diagnóstico por volta de $50 \%{ }^{40,42,43}$.

\section{f) Outros marcadores}

São diversas as pesquisas atuais em torno de substâncias que poderão constituir eficientes marcadores tumorais no câncer colorretal (telomerase ${ }^{44-50}$; $\mathrm{CD}_{4} 4^{51}$; metaloproteinase $^{52}$; c-erbB-2 ${ }^{53}$, E-caderina ${ }^{54}$, Bcl- $^{55}$ ), dosados no soro periférico ou nos tecidos do paciente. Estudos clínicos não demonstraram até o momento marcador perfeitamente eficaz; no entanto, é possível que o marcador ideal se encontre entre as substâncias em estudo.

Percebe-se tendência à análise de substâncias teciduais para determinação de conduta terapêutica mais adequada. No entanto, determinações teciduais apresentam as limitações de utilização referidas anteriormente.

Encontram-se disponíveis conjuntos para dosagem em soro periférico de CEA, CA 242, CA 19-9 e CA 72-4. Por imuno-histoquímica podem ser determinados no tecido: proteína p $53^{56}$, angiogênese, c-erbB-2, E-caderina, DCC e $\mathrm{Bcl}-2$, relevantes como marcadores tumorais. Estudos genéticos são possíveis para determinação de alterações, em casos de HNPCC (câncer colorretal hereditário não polipóide), por exemplo, mas de custo elevado e de disponibilidade reduzida atualmente.

\section{Momentos de Utilização dos Marcadores Séricos}

Os marcadores tumorais séricos devem ser determinados em diversos momentos no tratamento do câncer colorretal; dosagens isoladas não são de utilidade e podem levar a errôneas interpretações do quadro clínico.

Os marcadores podem ser analisados nas etapas de diagnóstico, estadiamento, determinação de prognóstico, avaliação de resposta ao tratamento e detecção de recidiva tumoral no período pós-operatório.

Os marcadores ainda não se demonstraram eficientes no rastreamento populacional de indivíduos portadores de neoplasias colorretais assintomáticos. A sensibilidade para diagnóstico dos marcadores atuais tem se revelado inferior a 50\%, inviabilizando tais métodos para finalidades diagnósticas 5 .

O uso de marcadores na determinação inicial do estadiamento, no entanto, demonstra-se efetivo e tem sido pouco utilizado. Entende-se, de forma geral, que a produção de substâncias marcadoras é proporcional às dimensões da massa tumoral apresentada pelo paciente. Nos pacientes cujo estadiamento clínico e cirúrgico demonstre estágios intermediários de desenvolvimento neoplásico (Dukes B ou C e Estádios II ou III da Classificação TNM) nível elevado inicial de marcadores tumorais pode indicar a presença de micrometástases e determinar opção por tratamento adjuvante (quimio ou radioterapia) ${ }^{57,58}$.

Não há correlação do nível inicial dos marcadores tumorais em relação ao grau de diferenciação de neoplasias colorretais ${ }^{28}$. Tumores altamente indiferenciados por vezes não produzem determinados marcadores, pela sua própria indiferenciação celular. 
No seguimento dos pacientes submetidos a tratamento pretensamente curativo a utilização dos marcadores se demonstra mais relevante ${ }^{12}$. Permite avaliar a eficiência do tratamento: níveis normais dos marcadores no pós-operatório mediato indicam provável radicalidade no tratamento, e persistência de níveis elevados significa caráter paliativo da terapêutica. Com elevada acurácia os marcadores são capazes de permitir a detecção precoce de recidivas tumorais assintomáticas e em estágio inicial nos casos submetidos a tratamento aparentemente curativo e radical ${ }^{10,11}$.

\section{Normas Atuais de Utilização de Marcadores Séricos}

A determinação em soro periférico do antígeno carcinoembrionário persiste como melhor método atual no que se refere à utilização de marcadores no câncer colorretal $^{7,59}$. Entretanto, o CEA não deve ser empregado como método de rastreamento populacional para identificação de portadores assintomáticos de neoplasias de cólon e de reto $^{59,60}$.

A elevação pós-operatória do antígeno carcinoembrionário, isoladamente, em pacientes submetidos a tratamento cirúrgico considerado radical, não autoriza a administração de métodos adjuvantes de tratamento; sinaliza a necessidade de emprego de métodos endoscópicos e radiológicos para identificação de possível recidiva em estágio inicial ${ }^{7,15,59}$.

Um aumento persistente de duas a três vezes o valor basal de CEA para o paciente significa elevada probabilidade de ocorrência de recidiva tumoral, exigindo exames adicionais e intensificação do seguimento ${ }^{60,61}$. A determinação do valor basal para o paciente no início do tratamento e verificações seriadas são fundamentais. Dosagens isoladas e análises baseadas no valor de referência de $5 \mathrm{ng} / \mathrm{ml}$ não constituem critérios adequados para determinação de normalidade.

O seguimento dos pacientes deve se estender durante sete anos a partir do tratamento inicial; em pacientes identificados à colonoscopia como formadores de pólipos o seguimento deve se prolongar além desse limite 5,60,61.

Novos marcadores ainda precisam ser comprovados como eficazes em estudos clínicos para recomendação de emprego rotineiro em pacientes ${ }^{59,60}$.

\section{Seguimento dos Pacientes e Marcadores}

O seguimento dos pacientes no período pós-operatório em tratamentos pretensamente curativos não é consensual.

É possível acompanhamento somente clínico dos pacientes ${ }^{62}$. Constitui opção a dosagem seriada de marcadores, com exames adicionais frente à elevação dos valores basais ${ }^{7,10,11}$. É possível submissão dos pacientes periodicamente a dosagens séricas, exames de imagem e procedimentos endoscópicos com o intuito de pronta detecção de recidiva tumoral ${ }^{61}$. Há controvérsias a respeito ${ }^{63-65}$.
Em nossa experiência ${ }^{16}$ não tem sido observado resultado eficaz no seguimento intensivo do paciente. Os marcadores tumorais séricos elevam-se três a seis meses antes da elucidação do sítio de recorrência. No entanto, quando os métodos de imagem ou endoscópicos demonstram a lesão está geralmente se mostra irressecável. Obtém-se sucesso quando se encontram tumores metacrônicos, então ressecados com radicalidade pelo estágio inicial em que se encontram. Para que as recidivas neoplásicas sejam abordadas curativamente é preciso que os marcadores e que os métodos de imagem correspondentes sejam aperfeiçoados para identificação da recorrência em estágio inicial de desenvolvimento e com reduzida extensão.

\section{Marcador Sérico Ideal}

O marcador sérico ideal deve ser específico, elevando-se somente em neoplasias colorretais; sensível, aumentando na presença de tumores em estágios iniciais. Todas as células tumorais devem produzi-lo, permitindo correlação com estadiamento e determinação de índices prognósticos; todos os tumores devem produzi-lo, impedindo a ocorrência de falsos resultados negativos.

Deve estar disponível à classe médica, ter custo acessível e ser exeqüível. O marcador ideal inexiste atualmente, sendo o CEA a substância que mais se aproxima das características enumeradas, apesar de suas deficiências inerentes ${ }^{7,15}$.

\section{Perspectivas Atuais e Futuras}

Os marcadores tumorais séricos atuais para o câncer colorretal apresentam acurácia superior somente em casos de doença avançada ${ }^{5}$. Heterogeneidade em sua produção por células tumorais, dentro da própria massa tumoral e entre indivíduos, permite erros de diagnóstico e de avaliação.

A sobrevida em cinco anos ultrapassa $80 \%$ nos pacientes portadores de neoplasias Estádio I (TNM), não atingindo $20 \%$ nos que apresentam doença avançada (Estádio IV $)^{66}$. A detecção de tumores em estágios iniciais é fundamental para a obtenção de bons resultados de tratamento. Inclusive na busca de recidivas tumorais o diagnóstico precoce é extremamente relevante ${ }^{10,11}$.

Marcadores teciduais precisos possibilitariam correlação com suscetibilidade da neoplasia a terapêuticas adjuvantes, fato ainda não estabelecido.

$\mathrm{Na}$ realidade, inexiste o marcador tumoral sérico ou tecidual ideal. A identificação de marcadores tumorais eficientes possibilitará o estabelecimento de aperfeiçoado patamar no tratamento dos pacientes portadores de neoplasias malignas colorretais. Enquanto a descoberta e o uso de novos marcadores encontram-se em desenvolvimento, o entendimento e a racional utilização dos marcadores disponíveis atualmente, pela classe médica, possibilitarão o alcance de resultados superiores na terapêutica dos tumores de cólon e de reto. 


\begin{abstract}
Colorectal cancer is a clinical entity of a persistent relevance in clinical practice and its early diagnosis is a determinant factor to obtain better therapeutic results. Tumor markers are helpful means for a better approach to individuals with such neoplasm. In the present review, the authors analyze the phases in which surgical-clinical treatment markers must be used: diagnosis, determination of tumor stage, establishment of prognosis and detection of recurrence. Current and future markers and the consensus on their use are discussed. Causal factors for errors in diagnosis with markers and perspectives of use are also presented.
\end{abstract}

Key Words: Tumor markers; Biological; Colorectal neoplasms; Colonic neoplasms; Rectal neoplasms; Carcinoembryonic antigen.

\section{REFERÊNCIAS}

1. Wilmink ABM. Overview of the epidemiology of colorectal cancer. Dis. Colon Rectum 1997; 40:483-93.

2. Hoel DG, Davis DL, Miller AB et al. Trends in cancer mortality in 15 industrialized countries, 1969-1986. J. Natl. Cancer Inst. 1992; 84:313-20.

3. Hurst R, Stamos M, Wilmoth G et al. Rectal carcinoma: are we making a difference? Am. Surg. 1996; 62:806-10.

4. Cutler SJ, Myers MH, Green SB. Trends in survival rates of patients with cancer. N. Engl. J. Med. 1975; 293:122-4

5. Hünerbein $\mathrm{M}$. The value of tumor markers in colorectal cancer. Cancer Res. 1998; 146:48-55.

6. Thomson DMP, Krupey J, Freedman SO et al. The radioimmunoassay of circulating carcinoembryonic antigen of the human digestive system. Med. Sci. 1969 , 64:161-7.

7. Carriquiry LA, Piñeyro A. Should carcinoembryonic antigen be used in the management of patients with colorectal cancer? Dis. Colon Rectum 1999, 42:921-29.

8. Schwartz MK. Colorectal carcinoma and carcinoembryonic antigen (CEA). Clin. Chem. 1980, 26:1213-20.

9. Grizzle WE, Manne U, Jhala NC et al. Molecular characterization of colorectal neoplasia in translational research. Arch. Pathol. Lab. Med. 2001, 125:91-8.

10. Steele Jr. G, Zamcheck N, Wilson R et al. Results of CEAinitiated second-look surgery for recurrent colorectal cancer. Am. J. Surg. 1980, 139:544-8.

11. Minton JP, Hoehn JL, Gerber DM et al. Results of a 400patient carcinoembryonic antigen second-look colorectal cancer study. Cancer 1985, 55:1284-90.

12. Lucha PA, Rosen L, Olenwine JA. et al. Value of carcinoembryonic antigen monitoring in curative surgery for recurrent colorectal carcinoma. Dis. Colon Rectum 1997, 40:145-9.

13. Wichmann MW, Miller C, LAU-Werner U et al. The role of carcinoembryonic antigen for the detection of recurrent disease following curative resection of large-bowel cancer. Langenbecks Arch. Surg. 2000, 385:271-5

14. Moura RMAM, Matos D, Galvão Filho MM et al. Value of CEA level determination in gallbladder bile in the diagnosis of liver metastasis secondary to colorectal adenocarcinoma. São Paulo Med. J. 2001, 119:110-3.

15. Destri GL, Greco S, Rinzivillo $\mathrm{C}$ et al.. Monitoring carcinoembryonic antigen in colorectal cancer: is it still useful? Jpn. J. Surg. 1998, 28:1233-6.
16. Kim SB. Estudo comparativo entre os marcadores tumorais séricos CA 242 e CEA pré-operatórios no adenocarcinoma colorretal, no seu estadiamento e no prognóstico de doentes submetidos ao tratamento cirúrgico. Tese (Doutorado em Medicina). São Paulo. Universidade Federal de São Paulo, Escola Paulista de Medicina, 2000, 94p.

17. Forones NM, Tanaka M, Matos D. Relationship between mesenteric and peripheral blood levels of CA 19-9 in patients with colorectal cancer. Int. J. Biol. Markers 2001, 16:27-30.

18. Fernández-Fernández L, Tejero E, Tieso A. Significance of CA 72-4 in colorectal carcinoma. Comparison with CEA and CA 19-9. Eur. J. Surg. Oncol. 1995, 21:388-90.

19. Nilsson O, Johansson C, Glimelius B et al.. Sensitivity and specificity of CA 242 in gastro-intestinal cancer. A comparison with CEA, CA 50 and CA 19-9. Br. J. Cancer 1992, 65:215-21.

20. Filella X, Molina R, Grau JJ et al. Prognostic value of CA 19.9 levels in colorectal cancer. Ann. Surg. 1992, 216:55-9.

21. Nakayama T, Watanabe M, Teramoto T et al. CA 19-9 as a predictor of recurrence in patients with colorectal cancer. J. Surg. Oncol. 1997, 66:238-43.

22. Guadagni F, Roselli M, Cosimelli M et al.. TAG-72 (CA 724 Assay) as a complementary serum tumor antigen to carcinoembryonic antigen in monitoring patients with colorectal cancer. Cancer 1993, 72:2098-106.

23. Engarås B, Kewenter J, Nilsson $\mathrm{O}$ et al. CEA, CA 50 and CA 242 in patients surviving colorectal cancer without recurrent disease. Eur. J. Surg. Oncol. 2001, 27:43-8.

24. Moll R, Franke WW, Schiller DL et al. The catalog of human cytokeratins: patterns of expression in normal epithelia, tumor and cultured cells. Cell 1982, 31:11-4

25. Bjorklund B. Tissue polypeptide antigen (TPA): biology, biochemistry, improved assay methodology, clinical significance in cancer and other conditions, and future outlook. Antibiot. Chemother. 1978, 22:16-31.

26. Correale M, Arnberg H, Blockx P et al. Clinical profile of a new monoclonal antibody-based immunoassay for tissue polypeptide antigen. Int. J. Biol. Markers 1994, 9:231-8.

27. Rydlander L, Ziegler E, Bergman T et al. Molecular characterization of a tissue-polypeptide-specific-antigen epitope and its relationship to human cytokeratin 18. Eur. J. Biochem. 1996, 241:309-14. 
28. Plebani M, Paoli M, Basso D et al. Serum tumor markers in colorectal cancer staging, grading, and follow-up. J. Surg. Oncol. 1996, 62:239-44.

29. Lindmark G, Bergström R, Pahlman L et al. The association of preoperative serum tumor markers with $\mathrm{Du}$ kes' stage and survival in colorectal cancer. Br. J. Cancer 1995, 71:1090-4.

30. Stigbrand T. The versatility of cytokeratins as tumor markers. Tumour Biol. 2001, 22:1-3.

31. Lane DP. On the expression of the 553 protein in human cancer. Molec. Biol. Reports 1994, 19:23-29.

32. Hamelin R, Laurent-Puig P, Olschwang $S$ et al. Association of p53 mutations with short survival in colorectal cancer. Gastroenterol. 1994, 106:42-8.

33. Porter PL, Gown AM, Kramp SG et al. Widespread p53 overexpression in human malignant tumours. Am. J. Pathol. 1992, 140:145-53.

34. Winter SF, Minna JD, Johnson BE et al. Development of antibodies against p53 in lung cancer patients appears to be dependent on the type of p53 mutation. Cancer Res. 1992, 52:4168-74.

35. Polge A, Bourgaux JF, Bancel E et al. p53 and follow-up of colorectal adenocarcinomas. Dig. Dis. Sci. 1998, 43:1434-42.

36. Bhatavdekar JM, Patel DD, Ghosh $\mathrm{N}$ et al. Coexpression of $\mathrm{Bcl}-2$, c-Myc, and p53 oncoproteins as prognostic discriminants in patients with colorectal carcinoma. Dis. Colon Rectum 1997, 40:785-90.

37. Shiota G, Ishida M, Noguchi $N$ et al. Circulating p53 antibody in patients with colorectal cancer: relation to clinicopathologic features and survival. Dig. Dis. Sci. 2000, 45:122-8

38. Hammel P, Soussi T. Le dosage serique des anticorps antip53: application au cancer colorectal. Rev. Med. Interne 2000, 21:167-73.

39. Ishigami SI, Arii S, Furutani $\mathrm{M}$ et al. Predictive value of vascular endothelial growth factor (VEGF) in metastasis and prognosis of human colorectal cancer. Br. J. Cancer 1998, 78:1379-84.

40. Hyodo I, Doi T, Endo H et al. Clinical significance of plasma vascular endothelial growth factor in gastrointestinal cancer. Eur. J. Cancer 1998, 34:2041-45.

41. Fujisaki K, Mitsuyama K, Toyonaga A et al. Circulating vascular endothelial growth factor in patients with colorectal cancer. Am. J. Gastroenterol. 1998, 93:249-52

42. Takeda A, Shimada H, Imaseki H et al. Clinical significance of serum vascular endothelial growth factor in colorectal cancer patients: correlation with clinicopathological factors and tumor markers. Oncol. Rep. 2000, 7:333-8.

43. Broll R, Erdmann H, Duchrow M et al. Vascular endothelial growth factor (VEGF) - a valuable serum tumour marker in patients with colorectal cancer? Eur. J. Surg. Oncol. 2001, 27:37-42.

44. Engelhardt M, Albanell J, Drullinsky P et al. Relative contribution of normal and neoplastic cells determines telomerase activity and telomere length in primary cancers of the prostate, colon, and sarcoma. Clin. Cancer Res. 1997, 3:1849-57.

45. Engelhardt M, Kumar R, Albanell J et al. Telomerase regulation, cell cycle and telomere stability in primitive hematopoietic cells. Blood 1997, 90:182-93.
46. Kim NW, Piatyszek MA, Prowse KR et al. Specific association of human telomerase activity with immortal cells and cancer. Science 1994, 266:2011-2015.

47. Engelhardt M, Drullinsky P, Guillem J et al. Telomerase and telomere length in the development and progression of premalignant lesions to colorectal cancer. Clin. Cancer Res. 1997, 3:1931-41.

48. Brown T, Aldous W, Lance R et al. The association between telomerase, p53, and clinical staging in colorectal cancer. Am. J. Surg. 1998, 175:364-6.

49. Okayasu I, Mitomi H, Yamashita K et al. Telomerase activity significantly correlates with cell differentiation, proliferation and lymph node metastasis in colorectal carcinomas. J. Cancer Res. Clin. Oncol. 1998, 124:444-9.

50. Yoshida K, Sugino T, Goodison S et al. Detection of telomerase activity in exfoliated cancer cells in colonic luminal washings and its related clinical implications. Br. J. Cancer 1997, 75:548-53.

51. Wielenga VJ, Van Der Neut R, Offerhaus GJ et al. CD 44 glycoproteins in colorectal cancer: expression, function and prognostic value. Adv. Cancer Res. 2000, 77:169-87.

52. Baker EA, Bergin FG, Leaper DJ. Matrix metalloproteinases, their tissue inhibitors and colorectal cancer staging. Br. J. Surg. 2000, 87:1215-21.

53. Porebska I, Harlozinska A, Bojarowski T. Expression of the tyrosine kinase activity growth factor receptors (EGFR, ERB B2, ERB B3) in colorectal adenocarcinomas and adenomas. Tumour Biol. 2000, 21:105-15.

54. Kimura $\mathrm{T}$, Tanaka $\mathrm{S}$, Haruma $\mathrm{K}$ et al. Clinical significance of MUC1 and E-cadherin expression, cellular proliferation, and angiogenesis at the deepest invasive portion of colorectal cancer. Int. J. Oncol. 2000, 16:55-64.

55. Bhatavdekar JM, Patel DD, Chikhlikar PR et al. Molecular markers are predictors of recurrence and survival in patients with Dukes B and Dukes C colorectal adenocarcinoma. Dis. Colon Rectum 2001, 44:523-33.

56. Yamaguchi A, Kurosaka Y, Fushida S et al. Expression of p53 protein in colorectal cancer and its relationship to short-term prognosis. Cancer 1992, 70:2778-84.

57. Wanebo HJ, Rao B, Pinsky CM et al. Preoperative carcinoembryonic antigen level as a prognostic indicator in colorectal cancer. N. Engl. J. Med. 1978, 299:448-51.

58. Moertel CG, Fallon JR, Go VW et al. The preoperative carcinoembryonic antigen test in the diagnosis, staging and prognosis of colorectal cancer. Cancer 1986, 58:603-10

59. Bast RC, Ravdin P, Hayes DF et al. 2000 update of recommendations for the use of tumor markers in breast and colorectal cancer: clinical practice guidelines of the American Society of Clinical Oncology. J. Clin. Oncol. 2001, 19:1865-78.

60. Trillet-Lenoir V, Freyer G. Du bon usage des marqueurs tumoraux dans les cancers colorectaux et mammaires. Les recommandations de l'American Society of Clinical Oncology (ASCO). Bull. Cancer 1997, 84:767-8.

61. Gardner B. Surveillance in the management of the cancer patient with special reference to breast and colon cancer. Am. J. Surg. 1997, 173:141-4. 
62. Moertel CG, Fleming TR, MacDonald JJ et al. An evaluation of carcinoembryonic antigen (CEA) test for monitoring patients with resected colon cancer. JAMA 1993 270:943-7.

63. Virgo KS, Vernava AM, Longo WE et al. Cost of patient follow-up after potentially curative colorectal cancer treatment. JAMA 1995, 273:1837-41.

64. Bruinvels DJ, Stiggelbour AM, Kievit J et al. Follow-up of patients with colorectal cancer. A meta-analysis. Ann. Surg. 1994, 219:174-82.

65. Secco GB, Fardelli R, Rovida $S$ et al. Is intensive followup really able to improve prognosis of patients with locar recurrence after curative surgery for rectal cancer? Ann. Surg. Oncol. 2000, 7:32-7.
66. Zinkin LD. A critical review of the classifications and staging of colorectal cancer. Dis. Colon Rectum 1983, 26:3743.

Endereço para correspondência:

Dr. Luís César Fernandes

Alameda Santos 211 Conj. 304 - Paraíso

01419-000 — São Paulo-SP

E-mail: luiscfernandes@terra.com.br 